\title{
Blockage-induced condensation controlled by a local reaction
}

\author{
Emilio N. M. Cirillo, ${ }^{1, *}$ Matteo Colangeli, ${ }^{2, \dagger}$ and Adrian Muntean ${ }^{3, \dagger}$ \\ ${ }^{1}$ Dipartimento di Scienze di Base e Applicate per l'Ingegneria, Sapienza Università di Roma, via A. Scarpa 16, I-00161, Rome, Italy \\ ${ }^{2}$ Gran Sasso Science Institute, Viale F. Crispi 7, 00167 L'Aquila, Italy \\ ${ }^{3}$ Department of Mathematics and Computer Science, Karlstad University, Karlstad, Sweden
}

(Received 15 August 2016; published 14 October 2016)

\begin{abstract}
We consider the setup of stationary zero range models and discuss the onset of condensation induced by a local blockage on the lattice. We show that the introduction of a local feedback on the hopping rates allows us to control the particle fraction in the condensed phase. This phenomenon results in a current versus blockage parameter curve characterized by two nonanalyticity points.
\end{abstract}

DOI: 10.1103/PhysRevE.94.042116

The effect of local perturbations of stationary states is a fascinating problem in statistical mechanics. At equilibrium, away from phase transitions, local perturbations typically induce local effects, whereas in nonequilibrium stationary states even global effects can be observed, for instance, on the stationary currents.

This phenomenon is well known for the totally asymmetric zero range process (ZRP) on the torus with time-independent and homogeneous rates $[1,2]$, where the local perturbation (hereafter called blockage) is the reduction of the rate at which a single defect site of the one-dimensional lattice is updated. If the blockage perturbation is small, then no effect persists in the large volume limit, computed by keeping constant the ratio between the number of particles and the volume of the lattice, and the macroscopic stationary current is unaffected. Instead, when the perturbation becomes larger, the current decreases as a result of the condensation of particles at the defect site. In particular, the current is found to depend nonanalytically on the strength of the blockage perturbation.

Condensation phenomena in zero range models have been thoroughly investigated in the recent literature (cf., e.g., Ref. [3], where a detailed analysis of the literature is provided). The effect of the blockage, on the other hand, has also been widely studied in the framework of the totally asymmetric simple exclusion process [4-6] and in its parallel counterpart [7]. These cases are particularly relevant since the behavior of the current cannot be explained in terms of the condensation, due to the imposed exclusion constraint. The main issue tackled there was, indeed, to understand whether the decrease of the current takes place as soon as the rate on the defect site is modified or, alternatively, only when a certain critical value of the strength of the blockage perturbation is reached. Related results have been also proved in Ref. [8]. It is worth mentioning that, in the recent literature, ZRPs with modified blockage rules have also been studied in different frameworks, e.g., non-Markovian processes and traffic models [9-12].

In this paper, we consider the totally asymmetric zero range model and investigate the possibility to compensate the blockage effect via a local feedback mechanism. This

\footnotetext{
*emilio.cirillo@uniroma1.it

${ }^{\dagger}$ matteo.colangeli@gssi.infn.it

${ }^{\ddagger}$ adrian.muntean@kau.se
}

is realized by keeping the rate on the defect site constant until the occupation number on that site reaches an a priori fixed activation threshold. For larger occupation numbers, the rate increases proportionally to the occupation number itself. We show, both numerically and with analytic arguments, that such a "local reaction" allows us to contrast the condensate formation, in that it maintains the particle fraction in the condensed phase constant for large values of the strength of the blockage perturbation. We also point out that, with such a mechanism, the current versus blockage strength curve exhibits two nonanalyticity points.

For the zero range models the idea of the activation threshold has been introduced in Refs. [13-15], where different interpretations, ranging from pedestrian dynamics to the thermodynamical theory of phase transitions, have been considered. As for the pedestrian motion interpretation, the results discussed in this paper can be rephrased as follows: particles are regarded as pedestrians moving on a lane, and the blockage corresponds to the presence of a bottleneck or to a lack of visibility (dark, smoke, etc.). In this context, particle condensation is seen as a severe pedestrian traffic jam caused by obstacles hindering the flow on the blocked spot. In this perspective, the feedback mechanism considered in this paper (cf. also Refs. [13,14]) can be interpreted as follows: when the number of pedestrians on the defect spot exceeds the "activation threshold" value, the ability of pedestrians to displace coherently increases because of the information exchange, which becomes significant as soon as the number of people on the spot is large enough. Thus, our results indicate that the jamming effect induced by the bottleneck can be compensated by an effective information exchange mechanism.

We now define the ZRP to be studied in this paper and borrow the notation from Ref. [2]. We consider the positive integers $L, N$, the finite torus $\Lambda=\{1, \ldots, L\}$, and the finite state or configuration space $\Omega_{L, N}$ made of the states $n=$ $\left(n_{1}, \ldots, n_{L}\right) \in\{0, \ldots, N\}^{\Lambda}$ such that $\sum_{x=1}^{L} n_{x}=N$. Given $n \in \Omega_{L, N}$ the integer $n_{x}$ is called number of particles at site $x \in \Lambda$ in the state or configuration $n$. The integer $1 \leqslant T \leqslant N$ and the real $0<q \leqslant 1$ are, respectively, called activation threshold and blockage parameter. Note that for $q$ close to one the strength of the blockage perturbation is small, whereas it is large for $q$ close to zero. For any site $x \in \Lambda$, the hopping rate $u_{x}: \mathbb{N} \rightarrow \mathbb{R}_{+}$is defined as follows: $u_{x}(0)=0$ for $x=$ $1, \ldots, L, u_{1}(k)=q$ for $1 \leqslant k \leqslant T$ and $u_{1}(k)=q(k-T+1)$ 
for $T+1 \leqslant k \leqslant N$, and $u_{x}(k)=1$ for $x=2, \ldots, L$ and $1 \leqslant k \leqslant N$. The ZRP considered in this context is the continuous time Markov process $n(t) \in \Omega_{L, N}, t \geqslant 0$, such that each site $x$ is updated with a rate $u_{x}\left(n_{x}(t)\right)$ and, once a site $x$ is chosen, a particle is moved to the neighboring site $x+1$ (recall that periodic boundary conditions are imposed).

Note also that when $q=1$ and $T=N$ the model reduces to the standard zero range process whose states can be mapped into those of the simple exclusion process. If $T=N$ and $q<1$ the site at $x=1$ is partially blocked. The effect of this kind of blockage is well known (see Refs. [1, Sec. V.1] and [2, Sec. 5.2]); here we investigate the case $q<1$ and $T<N$ and show that, because of the local feedback acting on site 1 , the system is able to react to the condensation effect.

It can be proven (see, e.g., Ref. [2, Eq. (15)]) that the invariant or stationary measure of the ZRP process is

$$
\mu_{L, N}(n)=\frac{1}{Z_{L, N}} \times \begin{cases}1 & \text { if } n_{1}=0 \\ 1 /\left[u_{1}(1) \cdots u_{1}\left(n_{1}\right)\right] & \text { otherwise }\end{cases}
$$

for any $n \in \Omega_{L, N}$, where the partition function $Z_{L, N}$ is the normalization constant

$$
\begin{aligned}
Z_{L, N}= & \sum_{k=0}^{T} q^{-k}\left(\begin{array}{c}
L+N-k-2 \\
N-k
\end{array}\right)+\sum_{k=T+1}^{N} \frac{q^{-k}}{(k-T+1) !} \\
& \times\left(\begin{array}{c}
L+N-k-2 \\
N-k
\end{array}\right) .
\end{aligned}
$$

The main results discussed in the sequel will be deduced in the thermodynamic limit $N, L \rightarrow \infty$, with $N / L=\rho$ being the global constant density and $T / N=\alpha$. The use of system-sizedependent hopping rates (cf. also Refs. $[16,17]$ ) is motivated here by the fact that we want to introduce the reaction effect as mildly as possible, in the sense that the local rate at site 1 starts to increase with the number of particles only if the local occupation number exceeds an amount proportional to $N$. At the end of the paper, we shall also comment on the dramatic effects observed if the threshold is chosen independent of $N$.

The main quantity of interest in our study is the stationary current representing the average number of particles crossing a bond between two given sites in unit time. More precisely, since periodic boundary conditions are imposed, the current does not depend on the chosen bond and is given by

$$
J_{L, N}=\mu_{L, N}\left[u_{x}\right]=Z_{L, N-1} / Z_{L, N} .
$$

The first equality defines the current, whereas the second one is proven in Ref. [2, Eq. (11)]. Another relevant quantity is the stationary particle fraction at the defect site $v_{L, N}=$ $\mu_{L, N}\left[n_{1}\right] / N$. When discussing the thermodynamic limit, we shall drop the subscripts $L$ and $N$ from the notation and write $J$ and $v$ for the stationary current and particle fraction at site 1 , respectively.

To evaluate the behavior of the partition function in the above limit, it is useful to introduce the function $I(k)$ by rewriting (2) as $Z_{L, N}=\sum_{k=0}^{N} \exp \{L I(k)\}$. To understand where the maxima of $I(k)$ are located, we express $I(k+1)-I(k)$ as

$$
I(k+1)-I(k)=\frac{1}{L}\left[\ln \frac{N-k}{(L+N-k-2) q}\right]
$$

for $0 \leqslant k \leqslant T-1$ and

$$
I(k+1)-I(k)=\frac{1}{L}\left[\ln \frac{N-k}{(L+N-k-2)(k-T+2) q}\right]
$$

for $T \leqslant k \leqslant N-1$. By using the two formulas above we can prove that, for large $L$, the function $I(k)$ has a single maximum attained in $k^{*}$, with $k^{*}=1$ for $q>q_{\rho}=\rho /(1+\rho)$, $k^{*}=L\lfloor(\rho-q(1+\rho)) /(1-q)\rfloor$ for $q_{\rho}>q>q_{\alpha}=\rho(1-$ $\alpha) /[1+\rho(1-\alpha)], k^{*}=\alpha N$ for $q_{\alpha}>q>q_{\alpha} / 2$, and $k^{*}$ is given by the smallest solution of the equation

$$
q[L(1+\rho)-k-2](k-\alpha \rho L+2)=\rho L-k
$$

for $q_{\alpha} / 2>q>0$. The explicit expression of $k^{*}$ in the latter case is rather lengthy and will be omitted here. The only property we rely on is the fact that, in the thermodynamic limit, $k^{*} / N$ tends to $\alpha$.

The computation of the partition function for the case $q>q_{\alpha}$ follows the scheme adopted in Ref. [2, Sec. 5.2]. Indeed, here the terms of the second sum in (2) can be neglected. In particular, for $q>q_{\rho}$ and $L$ large, by expanding the binomials in (2) for $k \sim O(1)$, one finds

$$
Z_{L, N}=\left(\begin{array}{c}
L+N \\
N
\end{array}\right) \frac{1}{1+\rho} \frac{q}{q(1+\rho)-\rho},
$$

which, using (3), yields $J=q_{\rho}$. Moreover, by computing the average occupation number on the defect site, one finds $\rho /(q(1+\rho)-\rho)$, so that in the thermodynamic limit the particle fraction $v$ at site 1 vanishes. Instead, if $q_{\alpha}<q<q_{\rho}$, the system undergoes condensation. In this case, using the Stirling's approximation and computing the resulting Gaussian integral, one finds

$$
Z_{L, N}=(1-q)^{-L-1} q^{-N} .
$$

Hence, (3) implies $J=q$ and, by computing the mean value of $n_{1}$, one obtains $v=1-q /[(1-q) \rho]$. Thus, in this particular regime, the particle fraction on the defect site is finite (i.e., condensation occurs) and the current is found to decrease linearly when $q$ decreases (i.e., the strength of the blockage perturbation increases).

To treat the case $0<q<q_{\alpha}$, one has to consider that, for large $L$, the function $I(k)$ attains its maximum at $\alpha N$. It is then useful to rewrite the partition function (2) by performing the changes of variables $h=T-k$ and $h=k-T$ in the first and in the second sum, respectively. Then one can expand the binomials for $h \sim O(1)$ to find

$$
\begin{aligned}
Z_{L, N}= & \frac{q^{-T}}{[1+\rho(1-\alpha)]^{2}}\left(\begin{array}{c}
L+N-T \\
N-T
\end{array}\right) \\
& \times\left[\sum_{h=0}^{T} \lambda^{h}+\sum_{h=1}^{N-T} \frac{1}{\lambda^{h}(h+1) !}\right],
\end{aligned}
$$

where we have set $\lambda=q / q_{\alpha}<1$, so that both of the two series are converging. This expression of the partition function allows us to compute the stationary current via (3), which leads to $J=q_{\alpha}$. Moreover, by computing the mean of the occupation number at site 1 and taking the thermodynamic limit, one obtains $v=\alpha$.

This result is the answer to our initial question: it shows that the local reaction term affecting the rate at the defect 


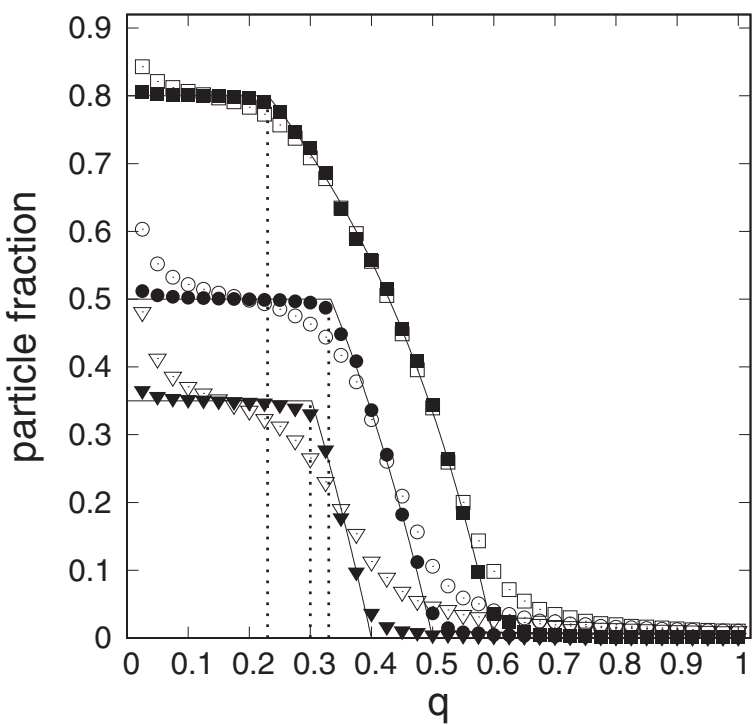

FIG. 1. Stationary particle fraction $v$ (at the defect site) vs $q$. Open and solid symbols refer to $L=100$ and $L=1000$, respectively. Circles, squares, and triangles refer, respectively, to $\rho=1$ and $\alpha=$ 0.5 ( $\circ$ and $\bullet, q_{\alpha}=0.33, q_{\rho}=0.50$ ), $\rho=1.5$ and $\alpha=0.8$ ( $\square$ and $\mathbf{\square}, q_{\alpha}=0.23, q_{\rho}=0.60$ ), and $\rho=2 / 3$ and $\alpha=0.35$ ( $\nabla$ and $\boldsymbol{\nabla}$, $\left.q_{\alpha}=0.30, q_{\rho}=0.40\right)$. Solid lines indicate the analytically predicted behavior in the thermodynamic limit. Dotted lines indicate the values of $q_{\alpha}$.

site balances, although it does not cancel, the effect of the blockage which originates the condensation. Note that, along this interplay between blockage and local reaction, the phenomenon of condensation is not inhibited: below the critical value $q_{\alpha}$, the particle fraction in the condensed phase stays constant and equal to $\alpha$. Moreover, for $0<q<q_{\alpha}$, the stationary current is also constant. This means that the behavior of the current versus the blockage parameter $q$ reveals two nonanalyticity points: one corresponds to the onset of condensation at $q=q_{\rho}$, while the second one, at $q=q_{\alpha}$, points out the value of the blockage parameter at which the reaction term becomes so effective to stop the rise of the particle fraction in the condensed phase.

Our analytical results are plotted in Figs. 1 and 2 together with the results of Monte Carlo simulations. The model has been simulated as follows: call $n(t)$ the configuration at time $t$, (1) a number $\tau$ is picked up at random with exponential distribution of parameter $\sum_{x=1}^{L} u_{x}\left(n_{x}(t)\right)$ and time is updated to $t+\tau$, (2) a site is chosen at random on the lattice with probability $u_{x}\left(n_{x}(t)\right) / \sum_{x=1}^{L} u_{x}\left(n_{x}(t)\right)$, and (3) a particle is then moved from that site to the neighboring site on the right. The results shown in the figures reveal a very good match between the analytical prediction and the numerical measures. We stress that the agreement improves when the lattice size $L$ increases. Therefore, the numerical simulations fully confirm our description of the main features of the model.

We recall that the threshold in the reaction term has been chosen proportional to $N$ to let the reaction effect be weak enough (the activation threshold diverges in the thermodynamic limit). Yet, by setting the threshold equal to a constant, the description of the model changes dramatically.

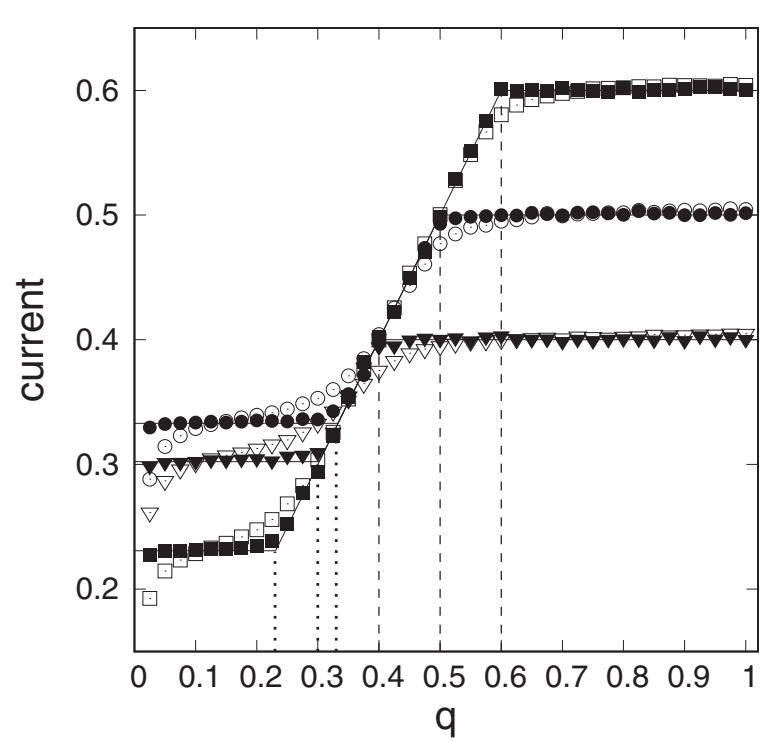

FIG. 2. Stationary current vs $q$. Symbols are as in Fig. 1. Dotted and dashed lines indicate, respectively, the values of $q_{\alpha}$ and $q_{\rho}$.

The Monte Carlo simulations plotted in Fig. 3 confirm that, in this case, the reaction term does inhibit the condensation. Indeed, the plot of the current versus the blockage parameter $q$ (scale on the right side of the bounding box) shows that the current is constant, namely, for any value of $q$ the current attains the value corresponding to the unperturbed dynamics [i.e., the dynamics with the hopping rates $u_{x}(k)=1$ for $x=1, \ldots, L$ and $1 \leqslant k \leqslant N]$. In this case no condensation is induced in the system, as can be seen by looking at the plot of the mean occupation number at the defect site (scale on the

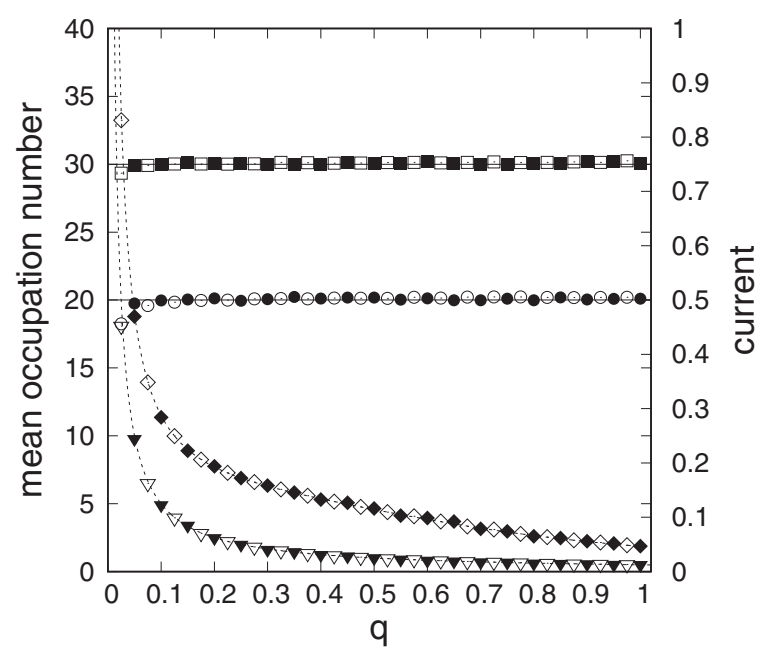

FIG. 3. Stationary occupation number at the defect site and current vs $q$. Open and solid symbols refer to $L=100$ and $L=1000$, respectively. Circles and squares denote the current at $\rho=1$ and $T=1$ ( $\circ$ and $\bullet$, value of the current 0.5 ) and at $\rho=3$ and $T=5$ ( $\square$ and $\mathbf{\square}$, value of the current 0.75 ), whereas triangles and diamonds denote the occupation number at site 1 at $\rho=1$ and $T=1$ ( $\nabla$ and $\nabla)$ and at $\rho=3$ and $T=5(\diamond$ and $\diamond)$, respectively. Solid and dotted lines indicate, respectively, the analytically predicted behavior of the current and the occupation number in the thermodynamic limit. 
left side of the bounding box). Note, indeed, that the mean occupation number at site 1 is of order one for any value of $q>0$; therefore the corresponding particle fraction $v$ tends to zero in the thermodynamic limit.

This occurs because the local feedback mechanism overwhelms the blockage effect and prevents condensation. In particular, the first sum in (2) is finite and can be estimated by expanding the binomial considering $k \sim O(1)$. For the second sum, after performing the change of variables $h=k-T$, one observes that, for large $L$, the sum concentrates on the terms $h \sim O(1)$, and, by accordingly expanding the binomials, one finds

$$
Z_{L, N}=\left(\begin{array}{c}
L+N \\
N
\end{array}\right) \frac{1}{(1+\rho)^{2}}\left[\frac{1-\sigma^{T+1}}{1-\sigma}+\sigma^{T-1}\left(e^{\sigma}-\sigma-1\right)\right]
$$

with $\sigma=q_{\rho} / q$. Hence, (3) yields $J=q_{\rho}$. By computing the average occupation number at site 1 , for $L$ large and $N=\rho L$, one obtains

$$
\begin{aligned}
& \frac{1}{\left(\begin{array}{c}
L+N \\
N
\end{array}\right)} Z_{L, N}(1+\rho)^{2} \mu_{L, N}\left[n_{1}\right] \\
& \sim \frac{\sigma}{(1-\sigma)^{2}}\left[-(T+1) \sigma^{T}(1-\sigma)+1-\sigma^{T+1}\right] \\
& \quad+(T-1) \sigma^{T-1}\left(e^{\sigma}-\sigma-1\right)+\sigma^{T}\left(e^{\sigma}-1\right)
\end{aligned}
$$

A comparison between numerical data and analytical prediction is given in Fig. 3, where the mean occupation number at site 1 was used in place of the particle fraction, because, as discussed above, the latter is a vanishing quantity.

\section{ACKNOWLEDGMENTS}

The authors thank E. Presutti, A. De Masi, B. Scoppola, D. Gabrielli, and C. Landim for many discussions and clarifying remarks.
[1] M. R. Evans, Braz. J. Phys. 30, 42 (2000).

[2] M. R. Evans and T. Hanney, J. Phys. A: Math. Gen. 38, R195 (2005).

[3] C. Godréche and J. M. Luck, J. Stat. Mech.: Theory Exp. (2012) P12012.

[4] S. A. Janowsky and J. L. Lebowitz, J. Stat. Phys. 77, 35 (1994).

[5] O. Costin, J. L. Lebowitz, E. R. Speer, and A. Troiani, Bull. Inst. Math. Acad. Sinica (New Series) 8, 49 (2013).

[6] S. A. Janowsky and J. L. Lebowitz, Phys. Rev. A 45, 618 (1992).

[7] B. Scoppola, C. Lancia, and R. Mariani, J. Stat. Phys. 161, 843 (2015).

[8] R. Basu, V. Sidoravicius, and A. Sly, arXiv:1408.3464v3.

[9] O. Hirschberg, D. Mukamel, and G. M. Schütz, Phys. Rev. Lett. 103, 090602 (2009).
[10] O. Hirschberg, D. Mukamel, and G. M. Schütz, J. Stat. Mech. Theory Exp. (2012) P08014.

[11] M. Cavallaro, R. J. Mondragón, and R. J. Harris, Phys. Rev. E 92, 022137 (2015).

[12] J. Kaupužs, R. Mahnke, and R. J. Harris, Phys. Rev. E 72, 056125 (2005).

[13] E. N. M. Cirillo, M. Colangeli, and A. Muntean, Comptes Rendus Mecanique 344, 19 (2016).

[14] E. N. M. Cirillo, M. Colangeli, and A. Muntean, Multiscale Model. Simul. 14, 906 (2016).

[15] E. N. M. Cirillo, M. Colangeli, and A. Muntean, J. Non-Equilib. Thermodyn. 41, 99 (2016).

[16] P. Chleboun and S. Grosskinsky, J. Phys. A: Math. Theor. 48, 055001 (2015).

[17] S. Grosskinsky and G. M. Schütz, J. Stat. Phys. 132, 77 (2008). 